\title{
Reaching optimal efficiencies using nanosized photoelectric devices
}

\author{
B. Rutten, ${ }^{1}$ M. Esposito, ${ }^{2, *}$ and B. Cleuren ${ }^{1, \dagger}$ \\ ${ }^{1}$ Hasselt University, B-3590 Diepenbeek, Belgium \\ ${ }^{2}$ Department of Chemistry and Biochemistry and Institute for Nonlinear Science, University of California-San Diego, \\ La Jolla, California 92093-0340, USA \\ (Received 10 August 2009; revised manuscript received 15 October 2009; published 16 December 2009)
}

\begin{abstract}
We study the thermodynamic efficiency of a nanosized photoelectric device and show that at maximum power output, the efficiency is bounded from above by a result closely related to the Curzon-Ahlborn efficiency. We find that this upper bound can be attained in nanosized devices displaying strong coupling between the generated electron flux and the incoming photon flux from the sun.
\end{abstract}

DOI: $10.1103 /$ PhysRevB.80.235122

PACS number(s): 05.70.Ln, 05.30.- d, 73.50.Pz

Understanding and controlling the mechanisms that determine the efficiency of photoelectric devices is of fundamental importance in the quest for efficient and clean sources of energy. Thermodynamically speaking, these devices are driven by the temperature difference between a hot reservoir (sun, temperature $T_{s}$ ) and a cold reservoir (earth, ambient temperature $T$ ). Therefore, like any heat engine, the efficiency at which the conversion of radiation into electrical energy takes place has a universal upper bound given by the Carnot efficiency ${ }^{1}$

$$
\eta_{c}=1-T / T_{s} .
$$

Although this result has fundamental theoretical implications, it is of poor practical use since it is only reached when the device is operating under reversible conditions. Hence the generated power, defined as the output energy divided by the (infinite) operation time, goes to zero. In realistic circumstances of finite power output, the efficiency will necessarily be below the Carnot limit due to irreversible processes taking place in the device. Another source of possible efficiency decrease are energy losses within the device for example due to nonradiative recombination of charge carriers. Since the operational parameters of the device are mostly determined in such a way that a maximum power output is obtained, Curzon and Ahlborn examined in 1975 the efficiency of a Carnot cycle with a finite cycling time and, using the endoreversible approximation, found an efficiency at maximum power $\eta_{c a}=1-\sqrt{T / T_{s}}{ }^{2}$ This result is remarkable since it does not depend on the specific details of the system, and thus the question of universality naturally arises. Recent works ${ }^{3-7}$ have indeed demonstrated that in the linear regime (small temperature differences, $\left.\eta_{c} \ll 1\right)$ the Curzon-Ahlborn efficiency is universal for so-called strongly coupled systems, where the heat and work producing fluxes are proportional. In these systems internal energy losses are absent, implying that the resulting efficiency is exclusively determined by the (unavoidable) irreversible processes occurring at finite power. Hence, at least in the linear regime, the CurzonAhlborn efficiency is indeed a universal upper bound, with a similar status as the Carnot efficiency. In the nonlinear regime, the efficiency at maximum power becomes device dependent but is again found to be highest for strongly coupled systems. Remarkably, it remains closely related to the Curzon-Ahlborn result. 6,7
While energy losses are almost unavoidable in the macroscopic world, new technological developments at the nanoscale open up the road to highly efficient devices. In thermoelectric research, it is well established that the use of lowdimensional, nanostructured devices significantly increases the efficiency. Such devices have a sharply peaked density of states, a prerequisite for a good thermoelectric. ${ }^{7-11}$ A similar tendency toward the development of nanostructured materials and even single nanosized devices also occurred in photovoltaic applications. ${ }^{12-17}$

In view of these recent developments in nonequilibrium thermodynamics as well as in nanotechnologies, we propose in this paper to investigate the performance of a single nanosized photoelectric device. The discrete nature of its energy levels is essential to provide strong coupling and, thus, high efficiencies. A detailed microscopic description of the device dynamics is presented, which allows for an exact analysis of the efficiency, far into the nonlinear regime. Our central result is that this device displays strong coupling when nonradiative recombination processes can be ignored. The corresponding efficiency at maximum power is then found to be very close to the Curzon-Ahlborn result. More generally, strong coupling provides a guiding principle in the quest for building high efficiency photoelectric devices. Experimentally, the degree of coupling can be determined by measurement of the Onsager coefficients.

The nanodevice we consider is composed of two single particle levels of energy $E_{l}$ and $E_{r}\left(>E_{l}\right)$, which define the bandgap energy $E_{g}=E_{r}-E_{l}$. We assume that Coulomb interactions prevent two electrons to be present at the same time in the device. As a result, the device is either empty $(0)$ or has one electron in level $E_{l}$ or $E_{r}$ with respective probabilities $p_{i}$ with $i \in\{0, l, r\}$. The device is connected with two leads $(l$ and $r)$. The left (right) lead can only exchange electrons with the level $E_{l}\left(E_{r}\right)$ as illustrated in Fig. 1. Such a nanodevice could be made for example of two coupled single-level quantum dots, each connected to a given lead. The leads are at the same temperature $T$ but have different chemical potentials $\mu_{l}$ and $\mu_{r}=\mu_{l}+q V$ due to an applied voltage ( $q$ is the electron charge). Electron transitions between $E_{l}$ and $E_{r}$ are induced by two possible mechanisms. The first is due to the incoming sun (black body) radiation at the resonant energy $h \nu=E_{g}$. The second is due to nonradiative processes at the same resonant transition. The dynamics of the cell is described using a master equation for- 


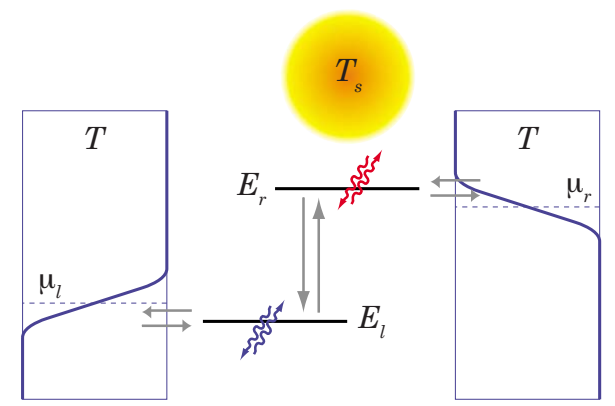

FIG. 1. (Color online) Schematic view of the nanosized photoelectric device. The gray arrows show the different allowed electron transitions. Transitions between the two energy levels are induced by solar photons (upper curved arrows, red) and by nonradiative processes (lower curved arrows, blue).

mulation for driven open systems ${ }^{6,18}$ presented below. Such a description can be shown to be equivalent to the quantum dynamics systematically derived from the microscopic Hamiltonian in Refs. 19 and 20 when the level broadening is smaller than $E_{g}$. The master equation reads

$$
\left[\begin{array}{c}
\dot{p}_{0}(t) \\
\dot{p}_{l}(t) \\
\dot{p}_{r}(t)
\end{array}\right]=\left[\begin{array}{ccc}
-k_{l 0}-k_{r 0} & k_{0 l} & k_{0 r} \\
k_{l 0} & -k_{0 l}-k_{r l} & k_{l r} \\
k_{r 0} & k_{r l} & -k_{0 r}-k_{l r}
\end{array}\right]\left[\begin{array}{c}
p_{0}(t) \\
p_{l}(t) \\
p_{r}(t)
\end{array}\right],
$$

where $k_{i j}$ denotes the transition rate from state $j$ to $i$. The rates describing the exchange of electrons with the leads are given by

$$
\begin{aligned}
k_{l 0}=\Gamma_{l} f\left(x_{l}\right), & k_{0 l}=\Gamma_{l}\left[1-f\left(x_{l}\right)\right], \\
k_{r 0}=\Gamma_{r} f\left(x_{r}\right), & k_{0 r}=\Gamma_{r}\left[1-f\left(x_{r}\right)\right],
\end{aligned}
$$

where $f(x)=[\exp (x)+1]^{-1}$ is the Fermi distribution. The arguments are the scaled energies $x_{l}=\left(E_{l}-\mu_{l}\right) /\left(k_{B} T\right)$ and $x_{r}$ $=\left(E_{r}-\mu_{r}\right) /\left(k_{B} T\right)$ with $k_{B}$ is the Boltzmann constant. The rates describing the transitions between energy levels due to nonradiative effects $(n r)$ and to sun photons $(s)$ are given by

$$
\begin{gathered}
k_{r l}=\Gamma_{n r} n\left(x_{g}\right)+\Gamma_{s} n\left(x_{s}\right), \\
k_{l r}=\Gamma_{n r}\left[1+n\left(x_{g}\right)\right]+\Gamma_{s}\left[1+n\left(x_{s}\right)\right],
\end{gathered}
$$

where $n(x)=[\exp (x)-1]^{-1}$ is the Bose-Einstein distribution with scaled energies $x_{g}=E_{g} /\left(k_{B} T\right)$ and $x_{s}=E_{g} /\left(k_{B} T_{s}\right)$. Notice that the ratio of the forward and backward transition rates associated to a given elementary process satisfies the detailed balance condition. This ensures that the equilibrium distribution (when $\mu_{l}=\mu_{r}$ and $T=T_{s}$ ) has the corresponding grandcanonical form. The electron current entering the device from the left lead is given by

$$
J=k_{l 0} p_{0}-k_{0 l} p_{l} .
$$

From now on, we will focus on the steady state dynamics of the device defined by $\dot{p}_{0}(t)=\dot{p}_{l}(t)=\dot{p}_{r}(t)=0 . J$ becomes the current of electrons through the device (positive from left to the right) with a corresponding electric current $q J$. It can be decomposed as $J=J_{s}+J_{n r}$ with $J_{s}$ and $J_{n r}$ the contributions to the current due to the interaction with the sun and the nonradiative processes, respectively:

$$
\begin{gathered}
J_{s}=\Gamma_{s} n\left(x_{s}\right) p_{l}-\Gamma_{s}\left[1+n\left(x_{s}\right)\right] p_{r}, \\
J_{n r}=\Gamma_{n r} n\left(x_{g}\right) p_{l}-\Gamma_{n r}\left[1+n\left(x_{g}\right)\right] p_{r} .
\end{gathered}
$$

From a thermodynamic viewpoint, solar cells are heat engines converting part of the heat input from the hot reservoir (the sun) into work by moving electrons from lower to higher chemical potentials. The remaining heat gets transferred to the colder reservoir (the earth). Since all photons interacting with the solar cell have an energy $E_{g}$, the net heat flux coming from the sun (i.e., the net energy absorbed per unit time) is $\dot{Q}_{s}=E_{g} J_{s}$. The heat flux coming from the cold reservoir has three contributions: $\dot{Q}_{l}=\left(E_{l}-\mu_{l}\right) J$ and $\dot{Q}_{r}$ $=-\left(E_{r}-\mu_{r}\right) J$ are due to electron exchanges between the cell and the left and right lead, respectively, and $\dot{Q}_{n r}=E_{g} J_{n r}$ is due to the non-radiative energy exchanges. The power $P$ generated by the solar cell to bring electrons from the left to the right lead is given by

$$
P=\left(\mu_{r}-\mu_{l}\right) J=T_{s}\left[x_{s}-\left(1-\eta_{c}\right)\left(x_{r}-x_{l}\right)\right] J .
$$

We verify that $P=\dot{Q}_{l}+\dot{Q}_{r}+\dot{Q}_{n r}+\dot{Q}_{s}$ since energy inside the cell is conserved at steady state. The efficiency at which this conversion takes place is then

$$
\eta=\frac{P}{\dot{Q}_{s}}=\frac{\left(\mu_{r}-\mu_{l}\right) J}{\left(E_{r}-E_{l}\right) J_{s}}=\left[1-\left(1-\eta_{c}\right) \frac{x_{r}-x_{l}}{x_{s}}\right]\left(1+\frac{J_{n r}}{J_{s}}\right) .
$$

We note that in practice, because not all incident light is absorbed by the device, this thermodynamic efficiency is higher than the photovoltaic efficiency defined as the ratio between the electrical power and the total incident light power. Our focus here is on the influence of the dynamical, irreversible processes taking place inside the device during the conversion of photonic energy into electrical power, and measured by $\eta$.

The entropy $S(t)$ of the solar cell can be expressed in the usual form $S(t)=-k_{B} \sum_{i} p_{i}(t) \ln p_{i}(t)$. Its time evolution can be separated in a reversible and irreversible part, $\dot{S}=\dot{S}_{e}+\dot{S}_{i}$, with $\dot{S}_{e}=\dot{Q}_{s} / T_{s}+\left(\dot{Q}_{l}+\dot{Q}_{r}+\dot{Q}_{n r}\right) / T$ corresponding to the entropy change due to the heat exchange with the different reservoirs and where $\dot{S}_{i} \geq 0$ can be identified as the internal entropy production due to dynamical processes within the solar cell. ${ }^{6,21,22}$ In the stationary regime $\dot{S}=0$ so that $\dot{S}_{i}=-\dot{S}_{e}$ and the entropy production takes on the familiar bilinear form

$$
\dot{S}_{i}=\dot{Q}_{s} \mathcal{F}_{U}+J \mathcal{F}_{N}=\left(x_{r}-x_{l}\right) J-x_{s} J_{s}-x_{g} J_{n r},
$$

where $\mathcal{F}_{U}=1 / T-1 / T_{s}$ and $\mathcal{F}_{N}=\left(\mu_{l}-\mu_{r}\right) / T$ are the thermodynamic forces conjugated to the energy and matter fluxes, respectively. Rearranging this expression leads to

$$
q V=\eta_{c} E_{g}\left(J_{s} / J\right)-T \dot{S}_{i} / J,
$$

which relates the work $q V$ done by the solar cell by moving a single electron up the potential gradient, to a fraction of the 
incident photon energy minus the irreversible losses. It reduces to the well known ideal cell formula $q V=E_{g} \eta_{c}$ when the device operates reversibly $\left(\dot{S}_{i}=0\right)$ and when $J_{s}=J$. This last condition implies that nonradiative recombination processes are absent, i.e., $J_{n r}=0$. Such an ideal situation was also considered by Shockley and Queisser in there seminal paper on the efficiency of $p-n$ junction solar energy converters. ${ }^{23}$ For our nano solar cell it means that the heat and particle flows are proportional, $\dot{Q}_{s}=E_{g} J$, a condition which is identified as thermodynamical strong coupling. ${ }^{3,6,24}$ For each electron transferred between the two leads, exactly one photon is involved. As we show below, this condition minimizes the entropy production and yields the maximal possible efficiency.

We start our analysis with a focus on the linear regime, close to thermal equilibrium. In this regime, characterized by small thermodynamic forces, the heat and particle flows appearing in the entropy expression (10) can be expanded to first order:

$$
\begin{gathered}
\dot{Q}_{s}=L_{U U} \mathcal{F}_{U}+L_{U N} \mathcal{F}_{N}, \\
J=L_{N U} \mathcal{F}_{U}+L_{N N} \mathcal{F}_{N} .
\end{gathered}
$$

The coefficients $L_{i j}$ appearing are the well-known Onsager coefficients. The off-diagonal elements are responsible for the energy conversion process, and satisfy the Onsager symmetry $L_{U N}=L_{N U}$. For a given temperature difference (quantified by $\mathcal{F}_{U}$ ), the power $P=-T \mathcal{F}_{N} J$ is maximal for $\mathcal{F}_{N}=$ $-\left(L_{N U} / 2 L_{N N}\right) \mathcal{F}_{U}$, which is (in the linear regime) exactly half the open circuit voltage (divided by the temperature $T$ ). The corresponding efficiency,

$$
\eta=\frac{\eta_{c}}{2} \frac{\kappa^{2}}{2-\kappa^{2}},
$$

is precisely half the Carnot efficiency multiplied by a factor

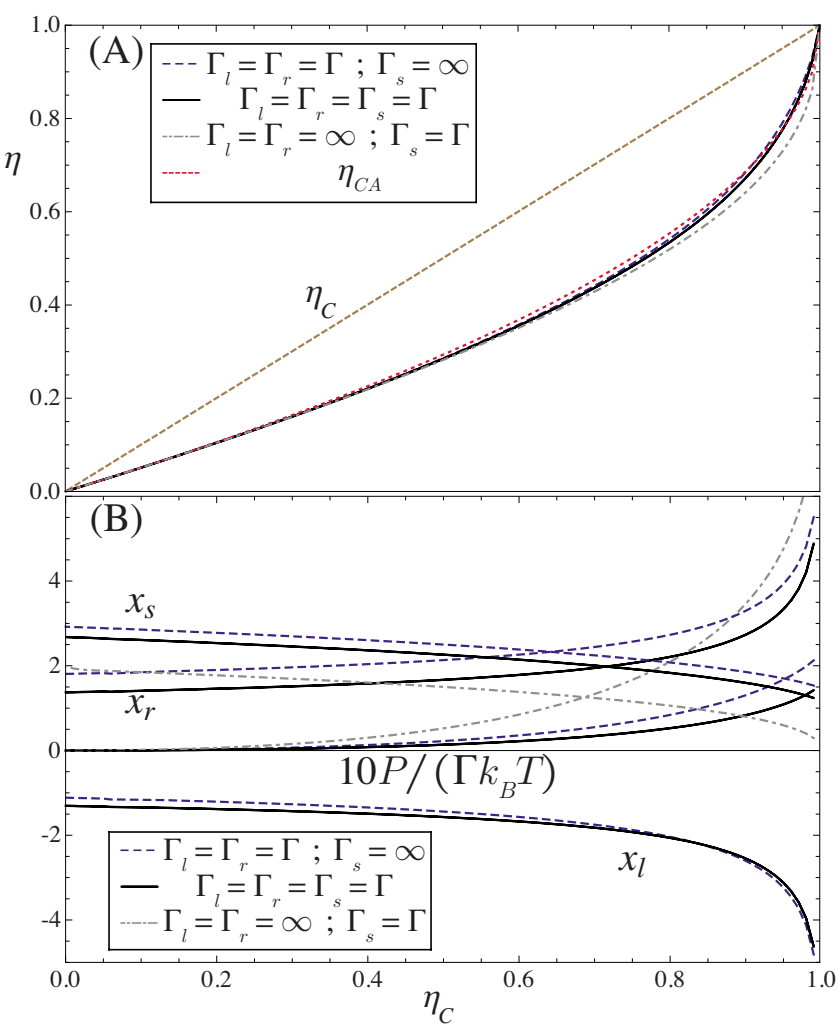

FIG. 2. (Color online) (a) The efficiency at maximum power in a strongly coupled device $\left(\Gamma_{n r}=0\right)$ as a function of $\eta_{c}$. (b) The corresponding values of the scaled energies $x_{l}, x_{r}$, and $x_{s}$. Results are given for different values of the coupling constants $\Gamma_{l}, \Gamma_{r}$, and $\Gamma_{s}$.

depending on the coupling parameter $\kappa=L_{U N} / \sqrt{L_{U U} L_{N N}}$ (Refs. 3 and 24) which has a numerical value between -1 and +1 since $\dot{S}_{i}$ must always be positive. Here, it is given by

$$
\kappa^{2}=\frac{e^{x_{l}}\left(e^{x_{g}}-1\right) \Gamma_{l} \Gamma_{r} \Gamma_{s}}{\left[\Gamma_{n r}\left(\Gamma_{l}+\Gamma_{r}\right)+e^{x_{l}}\left(\left(\Gamma_{n r}-\Gamma_{l}\right) \Gamma_{r}+e^{x_{g}} \Gamma_{l}\left(\Gamma_{n r}+\Gamma_{r}\right)\right)\right]\left(\Gamma_{n r}+\Gamma_{s}\right)} .
$$

As is clear from Eq. (13), the efficiency is maximal for $\kappa$ $= \pm 1$, corresponding to a strongly coupled system. From Eq. (14) this requires $\Gamma_{n r}=0$. Conversely, the entropy production at maximal power,

$$
\dot{S}_{i}=\mathcal{F}_{U}^{2} L_{U U}\left[1-(3 / 4) \kappa^{2}\right],
$$

reaches its minimal value in strongly coupled systems. We note that this reasoning is valid for any type of heat conversion device. And so, strong coupling can be used as a guiding principle in the development of highly efficient devices. As we demonstrate here, the use of nanosized devices provides an elegant solution to achieve this in practice.
We now extend our analysis to the nonlinear regime by using the full fledged analytical expressions for the various fluxes. For given values of $\eta_{c}$ and of $\Gamma$ 's, the maximum power output with respect to $x_{r}, x_{l}$, and $x_{s}$ cannot be found analytically. However, in the strong coupling case where $\Gamma_{n r}=0$ the numerical search for the maximum can be improved by carrying out some partial maximization analytically. Indeed, by defining $y=x_{r}-x_{l}$ and using Eq. (8), the condition $\partial_{y} P=0$ together with $\partial_{x_{x}} P=0$ implies the relation $\partial_{y} J=-\left(1-\eta_{c}\right) \partial_{x_{s}} J$, which can be solved analytically for $y$ as a function of $x_{s}$ (the cumbersome expression is not given here but can be derived using simple algebra). Using this solution in the expression for power implies that maximum power can 


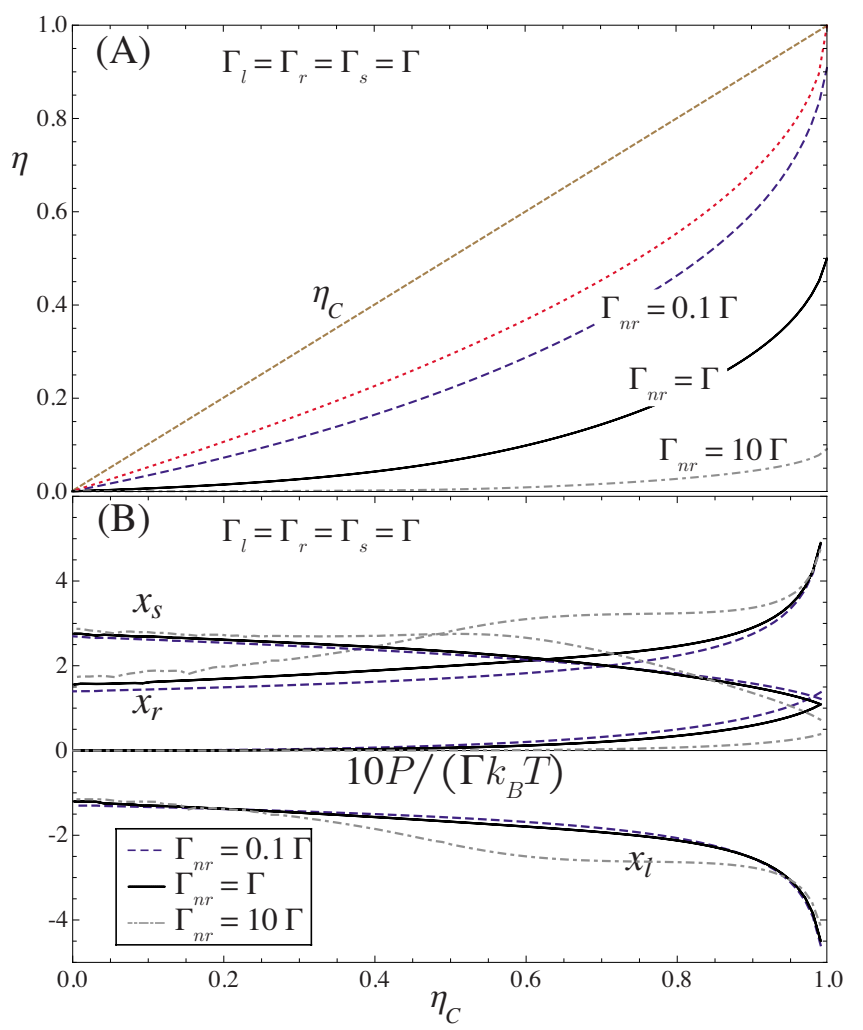

FIG. 3. (Color online) Same legend as Fig. 2 but for a device where nonradiative effects break down the strong coupling condition.

be obtained numerically by finding the maximum with respect to the two remaining variables $x_{s}$ and $x_{l}$. In the strong coupling regime, the results of the optimization are shown in Fig. 2. The efficiency at maximum power is plotted as a function of $\eta_{c}$ for different sets of $\Gamma$ 's, together with the corresponding values for $x_{l}, x_{r}$, and $x_{s}$ that maximize the power. The efficiency remains remarkably close to the Curzon-Ahlborn result for almost all values of $\eta_{c}$ and for the different sets of $\Gamma$ 's. Only slight deviations are observed far from equilibrium when $\eta_{c}$ is large. The appearance here of the Curzon-Ahlborn efficiency, therefore, goes beyond the usual endoreversible setup used to derive it. It points to a more fundamental character that can be traced back to the inherent presence of irreversible dynamics when the device is operating at maximum power. When, due to the presence
TABLE I. The efficiency at maximum power, together with the corresponding values for $E_{g}$ and $V$. In all cases we take $\Gamma_{l}=\Gamma_{r}$ $=\Gamma_{s}=\Gamma$.

\begin{tabular}{lccc}
\hline \hline & $\begin{array}{c}\eta \\
(\%)\end{array}$ & $\begin{array}{c}E_{g} \\
(\mathrm{eV})\end{array}$ & $\begin{array}{c}V \\
(\mathrm{~V})\end{array}$ \\
\hline$\Gamma_{n r} / \Gamma=0.0$ & 77.5 & 0.733 & 0.568 \\
$\Gamma_{n r} / \Gamma=0.1$ & 69.4 & 0.727 & 0.562 \\
$\Gamma_{n r} / \Gamma=1.0$ & 36.0 & 0.674 & 0.507 \\
$\Gamma_{n r} / \Gamma=10.0$ & 5.8 & 0.534 & 0.361 \\
\hline \hline
\end{tabular}

of nonradiative effects, the strong coupling condition is lost, the results in Fig. 3 show that the efficiency at maximum power is dramatically decreased below the Curzon-Ahlborn result. In the nonlinear regime, we where unable to find a region in the parameter space where the lack of strong coupling does not significantly reduce the efficiency. This supports the thesis that strong coupling is required in order to obtain the highest possible efficiency. Setting $\Gamma_{l}=\Gamma_{r}=\Gamma_{s}=\Gamma$, Table I summarizes the results obtained under practical conditions, i.e., by setting $T=295 \mathrm{~K}$ and $T_{s}=5780 \mathrm{~K}$ corresponding to $\eta_{c} \approx 95 \%$. An efficiency of $77.5 \%$ is obtained in the strong coupling case, which is slightly below the CurzonAhlborn result $\left(\eta_{c a} \approx 77.6 \%\right)$. Orders of magnitude remain the same when changing the relative values between the $\Gamma$ 's.

In summary, using a stochastic thermodynamics description of photo-electric devices, we have provided evidence that best efficiencies can be obtained with nano-sized cells which allow for a strong coupling between the photon flux from the sun and the electron flux through the device. In such devices Carnot efficiencies can be reached in the reversible limit where the power output goes to zero. In the situation of maximum power, which is of much greater practical interest, we found that the best efficiencies are remarkably well predicted by the Curzon-Ahlborn result. The presence of non-radiative effects needs to be avoided since it breaks down the strong coupling condition which leads to a drastic decrease in the efficiency.

M.E. is supported by the FNRS Belgium (chargé de recherches) and by the government of Luxembourg (Bourse de formation recherches). B.C. is supported by the FWOVlaanderen.
*Also at Center for Nonlinear Phenomena and Complex Systems, Université Libre de Bruxelles, Code Postal 231, Campus Plaine, B-1050 Brussels, Belgium.

†bart.cleuren@uhasselt.be

${ }^{1} \mathrm{H}$. B. Callen, Thermodynamics and an Introduction to Thermostatistics (John Wiley \& Sons, New York, 1985).

${ }^{2}$ F. Curzon and B. Ahlborn, Am. J. Phys. 43, 22 (1975).

${ }^{3}$ C. Van den Broeck, Phys. Rev. Lett. 95, 190602 (2005).

${ }^{4}$ A. Gomez-Marin and J. M. Sancho, Phys. Rev. E 74, 062102
(2006)

${ }^{5}$ C. Van den Broeck, Adv. Chem. Phys. 135, 189 (2007).

${ }^{6}$ M. Esposito, K. Lindenberg, and C. Van den Broeck, Phys. Rev. Lett. 102, 130602 (2009).

${ }^{7}$ M. Esposito, K. Lindenberg, and C. Van den Broeck, EPL 85, 60010 (2009).

${ }^{8}$ T. E. Humphrey and H. Linke, Phys. Rev. Lett. 94, 096601 (2005).

${ }^{9}$ L. D. Hicks and M. D. Dresselhaus, Phys. Rev. B 47, 16631 
(1993).

${ }^{10}$ G. D. Mahan and J. O. Sofo, Proc. Natl. Acad. Sci. U.S.A. 93, 7436 (1996).

${ }^{11}$ T. E. Humphrey, R. Newbury, R. P. Taylor, and H. Linke, Phys. Rev. Lett. 89, 116801 (2002).

${ }^{12}$ R. D. Schaller and V. I. Klimov, Phys. Rev. Lett. 92, 186601 (2004).

${ }^{13}$ V. I. Klimov, Appl. Phys. Lett. 89, 123118 (2006).

${ }^{14}$ D. Timmerman, I. Izeddin, P. Stallinga, I. N. Yassievich, and T. Gregorkiewicz, Nat. Photonics 2, 105 (2008).

${ }^{15}$ M. D. Kelzenberg, D. B. Turner-Evans, B. M. Kayes, M. A. Filler, M. C. Putnam, N. S. Lewis, and H. A. Atwater, Nano Lett. 8, 710 (2008).

${ }^{16}$ B. Tian, X. Zheng, T. J. Kempa, Y. Fang, N. Yu, G. Yu, J. Huang, and C. M. Lieber, Nature (London) 449, 885 (2007).

${ }^{17}$ B. Tian, T. J. Kempa, and C. M. Lieber, Chem. Soc. Rev. 38, 16 (2009).

${ }^{18}$ M. Esposito, U. Harbola, and S. Mukamel, Phys. Rev. E 76, 031132 (2007).

${ }^{19}$ M. Galperin and A. Nitzan, Phys. Rev. Lett. 95, 206802 (2005).

${ }^{20}$ M. Galperin and A. Nitzan, J. Chem. Phys. 124, 234709 (2006).

${ }^{21}$ J. Schnakenberg, Rev. Mod. Phys. 48, 571 (1976).

${ }^{22}$ L. Jiu-Li, C. Van den Broeck, and G. Nicolis, Z. Phys. B 56, 165 (1984).

${ }^{23}$ W. Shockley and H. J. Queisser, J. Appl. Phys. 32, 510 (1961).

${ }^{24}$ O. Kedem and S. R. Caplan, Trans. Faraday Soc. 61, 1897 (1965). 